\title{
The effect of a 12-week exercise intervention on the relationship between balance, age and physical activity
}

\begin{abstract}
Purpose: Prior studies have shown a correlation between increased sway scores and decreased balance in older adults. This has the potential to suggest an increased fall risk for those with impaired balance. Other studies have continued to measure decreased balance through a wide variety of testing techniques that occur with aging. The purpose of this study was to see if increasing physical activity could have a beneficial effect on balance scores in a population of faculty and staff at a large public university.
\end{abstract}

Methods: Nineteen adults between the ages of 28 and 65 participated in a 5 month exercise program. The program consisted of three times per week of organized exercise classes that were one hour in duration. Biodex sway scores were obtained during pre- and posttesting as an indicator of balance. Physical activity was measured through the use of a validated MOVband accelerometer that each individual wore on their wrist for the duration of the program. The MOVband used an algorithm to convert movement into miles for better analysis and understanding.

Results: There was a significant $(\mathrm{p}=0.022)$ decrease in sway scores from pre-testing (2.242) to post-testing (1.768) showing an increase in balance. Age was significantly correlated $(\mathrm{r}=0.765)$ with the change in sway score while controlling for miles. Pre-testing sway scores were also significantly correlated with age $(0.680)$. Post-testing sway scores were significantly negatively correlated with miles $(0.771)$. There was also a significant correlation between age and the change in sway score from pre- to post-testing $(0.644)$.

Conclusion: The correlation between age and pre-testing scores agrees with prior studies suggesting that as the individual ages, balance decreases. The negative correlation between post-testing sway scores and miles shows that increased physical activity has a positive effect on balance. This is also supported by the decreased sway scores from pre- to posttesting. Lastly, the correlation between age and change in sway score indicates that the older participants demonstrated an increased effect of physical activity on balance compared to the younger individuals. This continues to demonstrate the importance of physical activity in improving balance to decrease fall risk in the aging population.

Keywords: physical activity, biodex, movband
Volume 2 Issue 4 - 2015

\section{Curtis Fennell, Kylene Boka, Hayden Gerhart, Glickman E}

Department of Exercise Science, Kent State University, USA

\author{
Correspondence: Curtis Fennell, Department of Exercise \\ Science, Kent State University, 162 MACC Annex, 350 Midway \\ Drive Kent, OH, 44240, USA, Tel 330-672-2930, \\ Email cfennelI@kent.edu
}

Received: February 24, 2015 | Published: April 22, 2015

\section{Introduction}

Balance is an important component of health in order to maintain functional movement and prevent falls. It has been well documented in previous research that balance decreases with age. ${ }^{1}$ Among individuals aged 65 and older, one third experience a fall at least once per year. ${ }^{2,3}$ In 2012 the rate of nonfatal fall injuries receiving medical attention in the United States was 43 per 1,000 populations. This rate increased with age when adults were aged $\geq 18$ years and the rate for those aged $\geq 75$ years was 121 per 1,000 , a higher rate compared to all other age groups. ${ }^{4}$ The number of injurious falls increases with age. ${ }^{5,6}$ These falls can lead to serious consequences, including fractures, head trauma, lacerations and can lead to immobility or death., ${ }^{2,7}$

Participation in exercise, which includes balance training, results in improvements of balance and reduces the risk of falls and the fear of falling. ${ }^{8,9}$ The aging process leads to a reduction in physical activity, which results due to a variety of common issues and limitations. ${ }^{10}$ Some of these include physical disabilities, mental health factors and even altered social and motivational factors. Only about $22 \%$ of individuals above the age of 65 participate in regular physical activity and fewer than $11 \%$ above 85 years $^{11}$ engage in physical activity. As aging ensues there is loss of joint strength, ${ }^{12}$ reduced bone and muscle mass ${ }^{13}$ and neuromuscular decline. ${ }^{14}$ Research has demonstrated that individuals with higher bone mass and more robust structure of bones experience fewer falls. ${ }^{15}$. Since aging is associated with less physical activity and less physical activity may elicit loss of functional ability and weakening of joints, muscles and bones there is a need for aging individuals to continue to participate in regular physical activity.

Tai-chi, ${ }^{16}$ exergaming ${ }^{17}$ and cognitive-motor balance training ${ }^{18}$ have recently been evaluated as potential exercise modalities to reduce fall risk in aging populations. Garber et al. ${ }^{9}$ concluded that there may be balance benefits for individuals participating in physical activities requiring agility, balance and other motor skills. However, the optimal style and type of training to improve balance is still unclear for adults of all ages (or across the lifespan). ${ }^{9,19}$ Therefore, the purpose of this study was to investigate the effect of consistent physical activity through the use of supervised exercise classes on balance in younger and older adults. The hypothesis was that age would correlate with reduced balance. Additionally, the age would correlate with the greatest improvements in balance over the 5 month exercise program. 
Furthermore, researchers predicted physical activity to be correlated with the greatest improvements in balance.

\section{Methods}

Nineteen adult participants (50 111 years, range $28-65$ years) enrolled in a 5 month exercise program for faculty and staff at a large Midwest university. The program consisted of 60 minutes of organized exercise classes three times per week (Monday, Wednesday and Friday). Each day the participants selected one of the offered exercise classes: running, walking, "cardio dance", exercise with weights, or "boot camp". Participants selected one class per day with the option to switch between the classes throughout the program. All classes were taught and supervised by a trained certified exercise research staff from the exercise physiology department at the university. Static balance was assessed using the validated Biodex Postural Stability Test ${ }^{20}$ prior to beginning the exercise classes and at the end of the 5 month program. Subjects stood on the Biodex platform under three conditions: eyes open hard surface, eyes closed hard surface, eyes open soft surface, eyes closed soft surface. The Overall Stability Index was used to compare the amount of sway in the participant's postural stability during pre-testing and post-testing. Physical activity was assessed through the use of the validated MOVband accelerometer which each participant wore on the wrist for the duration of the program. The MOVband is a tri-axial accelerometer that measures movement in three planes and has been previously validated against the Acti-graph accelerometer. ${ }^{21}$ It measures movement in "moves" which can be converted to miles or steps. The data was analyzed with a paired samples t-tests for Biodex sway scores to examine the improvement from pre-testing to post-testing. Correlations for age and change in sway score were determined both with and without controlling for miles.

\section{Results}

The Overall Stability Index for all the participants at pre-testing was $2.24 \pm 0.63$. At post-testing, this index decreased to $1.77 \pm 0.70$, indicating an improvement in balance as the amount of sway detected significantly decreased as seen in Figure $1(\mathrm{p}=0.02)$. Correlation analysis indicated a significant correlation between pre-testing sway scores and age show in Figure $2(r=0.680)$. This data indicates that the older participants had higher sway scores, representing worse balance, than the younger participants at pre-testing. There was also a significant correlation $(\mathrm{r}=0.765)$ between age and the change in sway score both with and without controlling for physical activity in miles (Figure 3). The older participants had more improvement in balance scores over the course of the 5 month program than the younger participants. Lastly, Figure 4 shows a significant negative correlation $(\mathrm{r}=0.771)$ between post-testing sway scores and physical activity in miles. This correlation indicates that the more physically active the participants were the lower their sway score, indicative of better balance.

\section{Discussion}

This study found that balance decreases with age, as it is associated with improvements in balance and physical activity was correlated with enhanced balance. Previous studies have demonstrated that balance decreases with aging. ${ }^{1}$ The data presented here supports this by finding a positive correlation between sway scores and age of the participants. This has implications in fall risk for aging adults which is important to prevent injury. ${ }^{2-7}$ The correlation between age and change in sway scores showed that the older adults had a greater improvement in balance from pre-testing to post-testing compared to the younger volunteers. This suggests that engaging in physical activity may improve balance among older adults. This data can be used to support balance programs to minimize the fall risk across the lifespan.

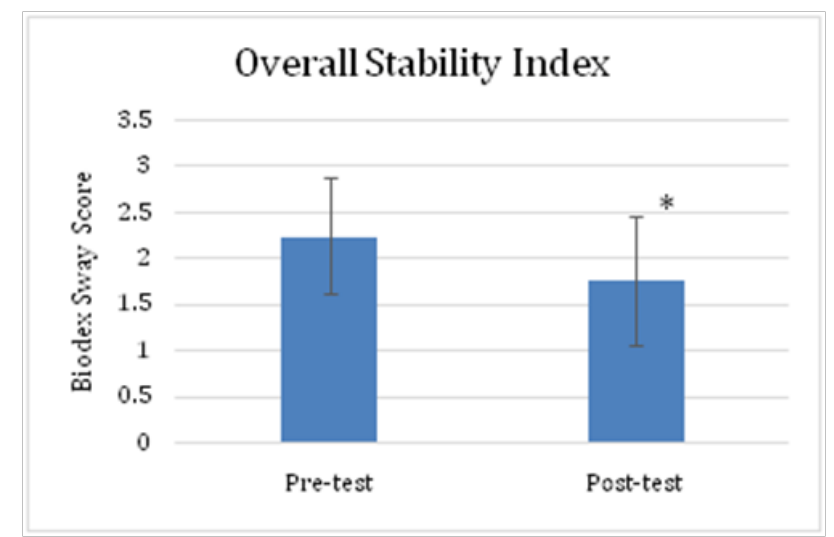

Figure I There was a significant improvement in the sway score for all participants from pre-testing to post-testing $(p=0.02)$.

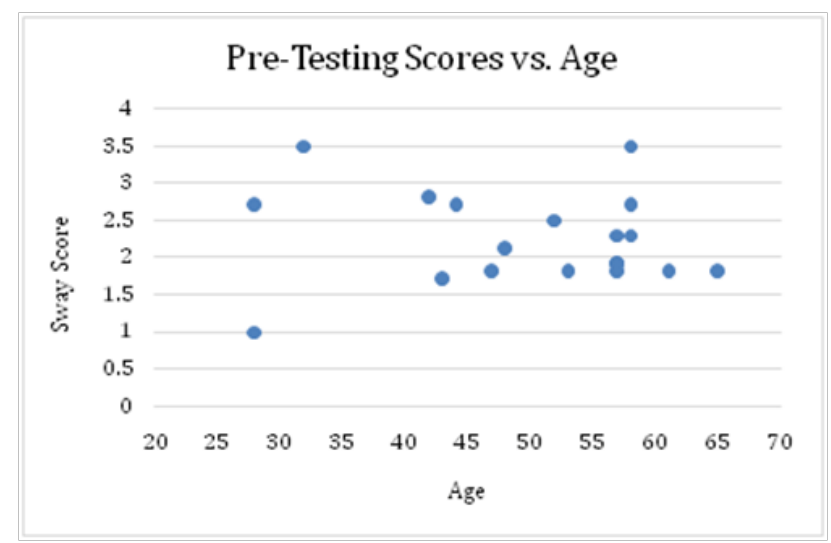

Figure 2 There was a significant correlation between pre-testing sway scores and age $(p=0.680)$.

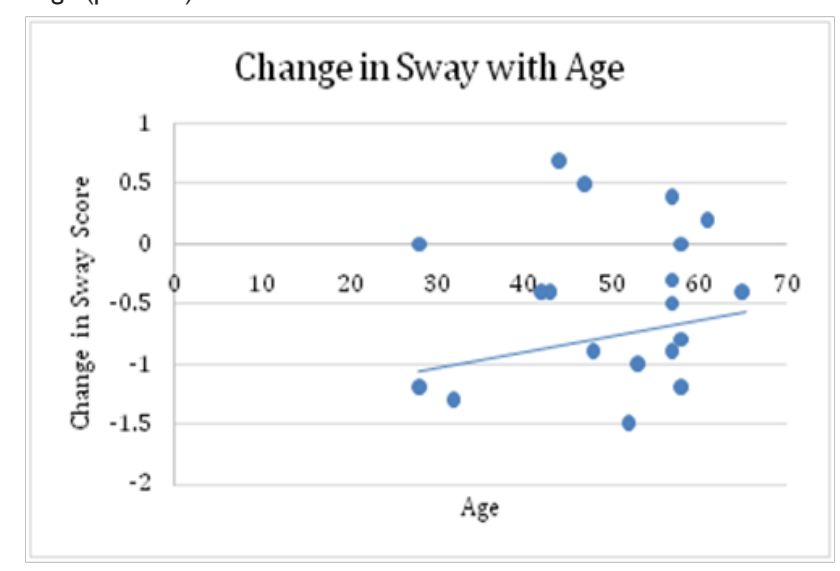

Figure 3 There was a positive correlation between the change in sway score and age without controlling for miles $(r=0.765)$.

Garber et al. ${ }^{9}$ and Nelson et al. ${ }^{19}$ previously concluded that there is not an optimal balance training plan for all ages. This investigation evaluated the use of consistent physical activity to improve balance 
in both young and older adults. The decreased sway scores from pre-testing to post-testing suggest that physical activity can have a significant effect on improving balance. In addition, the negative correlation in the post-testing sway scores and physical activity in miles demonstrated the influence physical activity can have on balance. This data supports prior studies that have demonstrated the benefit of physical activity on balance. ${ }^{89}$ However, this investigation elucidated the age-related effects of physical activity on balance. There was a greater improvement in balance in the older adult group than in the younger adult group. This suggests the importance of physical activity across the lifespan to prevent the age-related declines in balance and reduce the growing fall risk that is accompanied with age. A majority of falls in the older adult may be caused by impaired postural control and muscle and bone weakness. Regular physical activity at least 2 times per week helps to improve these weaknesses. ${ }^{89}$ This data demonstrated that physical activity is correlated with balance, thus physical activity may have improved balance by strengthening muscles and bones.

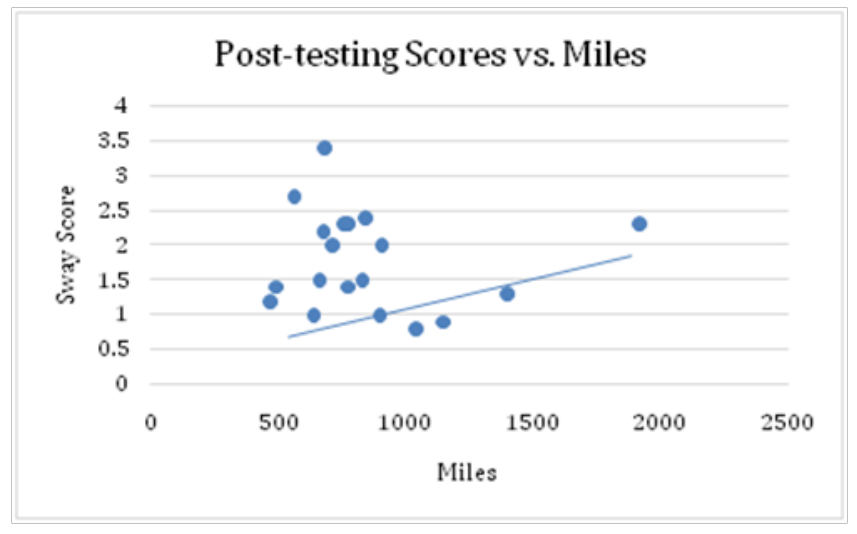

Figure 4 There was a significant negative correlation between post-testing scores and physical activity during the exercise program $(0.77 \mathrm{I})$.

\section{Summary}

This investigation supports the benefit a of consistent physical activity program on balance. It also evaluated the relationship between age and balance. There was significant improvement in balance at the end of the 5- month exercise program for all individuals. Increased age was related to a greater improvement in balance at the end of the consistent exercise program. This data supports the importance of exercise for all aging adults across the lifespan, to minimize the declining changes in balance. Future research should focus on interventions for an elderly the older adult to improve balance and functional movement.

\section{Reference}

1. Konrad HR, Girardi M, Helfert R. Balance and Aging. Laryngoscope. 1999;109(9):1454-1460.

2. Tinetti ME, Speechley M, Ginter SF. Risk factors for falls among elderly persons living in the community. N Engl J Med. 1988;319(26):17011707.

3. Campbell AJ, Borrie MJ, Spears GF. Risk factors for falls in a community-based prospective study of people 70 years and older. $J$ Gerontol. 1989;44(4):M112-117.
4. Adams P, Martinez ME, Kirzinger WK. Quick Stats: Rate of Nonfatal Fall Injuries Receiving Medical Attention, by Age Group - National Health Interview Survey, United States, 2012. Center for Disease Control and Prevention. 2014;63(29):641.

5. Rubenstein LZ. Falls in older people: epidemiology, risk factors and strategies for prevention. Age Ageing. 2006;35(suppl 2):ii37-ii41.

6. Tinetti ME, Williams CS. Falls, injuries due to falls and the risk of admission to a nursing home. $N$ Engl J Med. 1997;337(18):1279-1284.

7. Sterling DA, O’Connor JA, Bonadies J. Geriatric falls: injury severity is high and disproportionate to mechanism. J Trauma. 2001;50(1):116-119.

8. Chodzo-Zajko WJ, Proctor DN, Fiatarone Singh MA, et al. American College of Sports Medicine position stand. Exercise and physical activity for older adults. Med Sci Sports Exerc. 2009;41(7):1510-1530.

9. Garber CE, Blissmer B, Deschenes MR, et al. American College of Sports Medicine Position Stand. The quantity and quality of exercise for developing and maintaining cardiorespiratory, muscoskeletal and neuromotor fitness in apparently healthy adults: guidance for prescribing exercise. Med Sci Sports Exerc. 2011;43(7):1334-1359.

10. Sallis J, Bowles H, Bauman A, et al. Neighborhood environments and physical activity among adults in 11 countries. Am J Prev Med. 2009;36(6):484-490.

11. Federal Integragency Forum on Aging-Related Statistics (US). Older Americans2004; Key Indicators of Well-Being. Washington (DC): Federal Interagency Forum on Aging-Related Statistics. USA, pp. 1-159.

12. Araújo CGS. Flexibility assessment: normative values for flexitest from 5 to 91 years of age. Arq Bras Cardiol. 2008;90(4):257-263.

13. Palmer IJ, Runnels ED, Bemben MG, et al. Muscle-bone interactions across age in men. 2006;5(1):43-51.

14. Valdez G, Tapia JC, Kang H, et al. Attenuation of age-related changes in mouse neuromuscular synapses by caloric restriction and exercise. Proc Natl Acad Sci U S A. 2010;107(33):14863-14868.

15. Nikander R, Sievänen H, Uusi-Rasi K, et al. Loading modalities and bone structures at nonweight-bearing upper extremity and weightbearing lower extremity: A pQCT study of adult female athletes. Bone. 2006;39(4):886-894.

16. Jahnke R, Linda L, Rogers C, et al. A Comprehensive Review of Health Benefits of Qigong and Tai Chi. Am J Health Promot. 2010;24(6):e1-e25.

17. Laufer Y, Gali D, Kodesh E. Does a Wii-based exercise program enhance balance control of independently functioning older adults? A systematic review. Clin Interv Aging. 2014;9:1803-1813.

18. Schoene D, Valenzuela T, Lord SR, et al. The effect of interactive cognitive-motor training in reducing fall risk in older people: a systematic review. BMC Geriatr. 2014;14:107.

19. Nelson ME, Rejeski WJ, Blair SN, et al. Physical activity and public health in older adults: recommendation from the American College of Sports Medicine and the American Heart Association. Med Sci Sports Exerc. 2007;39(8):1435-1445.

20. Arnold BL, Schmitz RJ. Examination of Balance Measures Produced by the Biodex Stability System. J Athl Train. 1998;33(4):323-327.

21. Barkley J, Rebold M, Carnes A, et al. The validity of a commerciallyavailable, low-cost, wrist-mounted accelerometer during treadmill exercise and free-living physical activity. J Sports Sci. 2019;7(7):735740. 